\title{
5 Héroïnes féminines et femmes héroïques dans l'œuvre théâtrale de Rachel de Queiroz: Maria Bonita et Maria do Egito
}

Giulia Manera*

* Giulia Manera est doctorante à l'Université Paris Ouest Nanterre La Défense, où elle prépare une thèse en cotutelle avec l'USP (Brésil) sur les femme écrivaines dans le «romance de $30 »$ au Brésil. Formée en Lettres et Philosophie à l'Université de Bologne (Italie) et en Etudes Romanes-Portugais à Paris Ouest, elle Rente ses activités de recherche otamment vers l'analyse de l'écriture éminine et de la représentation du fémini dans la littérature brésilienn contemporaine.
Résumé : Dans la décennie de 1950 Rachel de Queiroz, qui à l'époque est désormais une écrivaine et une journaliste affirmée, se dédie aussi à l'écriture scénique. Elle compose deux pièces, Lampião (1953) et A Beata Maria do Egito (1957), qui se construisent autour de ses protagonistes féminines, : le personnage historique de Maria Bonita et la figure, appartenant à l'hagiographie chrétienne, de la Sainte Marie d'Egypte. Pour créer les deux Maria, la bandit et la sainte, les rendre cohérentes avec sa figuration du féminin et lui donner une individualité sur la scène, Rachel de Queiroz opère sur la matière régionale, historique et religieuse. La lecture de ce processus d'écriture permet non seulement d'analyser la représentation du féminin dans l'œuvre théâtrale de Rachel de Queiroz, mais aussi de comprendre en quelle manière cette représentation intègre la dramaturgie brésilienne contemporaine.

Mots-clés : théâtre; féminin; Rachel de Queiroz.
Resumo: Na década de 1950, Rachel de Queiroz, já uma escritora e uma jornalista renomada, começa a escrever para o teatro. Ela compõe duas peças, Lampião (1953) e A Beata Maria do Egito (1958), centradas nas suas protagonistas femininas: a personagem histórica de Maria Bonita e a figura da hagiografia cristã de Santa Maria do Egito. Para criar as duas Marias, a cangaceira e a beata, e lhe dar uma individualidade na cena, Rachel de Queiroz desenvolve a matéria regional, histórica e religiosa. A leitura deste processo de escritura permite não somente analisar a representação do feminino na obra cênica de Rachel de Queiroz, mas também compreender como esta representação dialoga com a dramaturgia brasileira contemporânea.

Palavras-chave: teatro; feminino; Rachel de Queiroz 
1 En 2010, le centenaire de Rachel de Queiroz a été commémoré dans tout le Brésil avec nombreux articles de presse, colloques et publications. A cette occasion, l'Institut Moreira Salles, qui conserve auss les archives les plus complètes de l'ous es archives les plus complètes de l'œuvre de l'auteur, a organisé l'exposition et les journées des conférences Rachel de Queiroz Centenária et publié le volume de poème inédits Mandacuru. L'année 2010 a été donc l'année de Rachel de Queiroz, une période pendant laquelle ses œuvres et son personnage ont attiré une attention

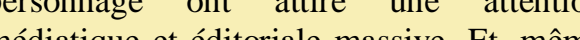
médiatique et editoriale massive. Et, mêm si l'ensemble de ces initiatives n'arriven pas vraiment à contribuer d'une façon consistante et novatrice au corpus critique de Rachel de Queiroz, une telle médiatisation semble témoigner, plus de dix ans après sa mort, de la vitalité de son œuvre et de l'importance de son héritage dans le panorama de la littérature cér le panorama de la litterature brésilienne contemporaine. A l'exception notable de son œuvre théâtrale qui, même en cette occasion mémorable, ne semble pas avoir bénéficié d'une reédition ni d'une nouvelle mise en scène.

2 Une pratique que l'auteur tient à revendiquer. Pendant l'entretien As Três Rachéis, pour le volume de Cadernos de I'Institut Moreira Salles qui lui est dédie, l' Rachel de Queiroz affirme : «Eu tenho dito que me sinto mais jornalista do que ficcionista. Sempre. Na verdade, minha profissão é essa: jornalista. Há cinquenta e tantos anos que todas as semanas eu escrevo pelo menos um artigo" (1997, p. 33).
« Minhas mulheres são danadas, não são? Talvez seja o ressentimento do que não sou e gostaria de ser » Rachel de Queiroz

\section{Rachel de Queiroz en scène}

Quand, en 1977, Rachel de Queiroz devient la première femme à entrer à l'Académie Brésilienne de Lettres, elle est élue "mais como escritora, no sentido genérico da palavra, do que como romancista" (MARTINS, 1997, p. 69). Si elle est donc reconnue essentiellement comme auteur de chroniques, et moins comme auteur de romans, son écriture théâtrale semble être oubliée, ou considérée non significative dans l'économie de sa production littéraire. La Rachel de Queiroz dramaturge est donc, finalement, considérée comme mineure. A l'appui de cette affirmation, on peut considérer que ses pièces semblent être rarement citées et encore plus rarement représentées, même à l'occasion des célébration et des nouvelles éditions pour le centenaire de sa naissance, en $2010^{1}$.

La lecture des pièces de Rachel de Queiroz - Lampião de 1953 et A Beata Maria do Egito de 1957 - révèle toutefois non seulement une écriture parfaitement cohérente et représentative de la poétique de l'écrivaine mais, dans une perspective plus large, permet aussi de reconnaître une figuration du féminin particulièrement significative et innovante dans la dramaturgie des années cinquante, une dramaturgie qui mérite une analyse attentive.

Bien que Rachel de Queiroz n'écrive pour la scène que de façon épisodique, notamment si on considère sa pratique presque quotidienne de l'écriture journalistique ${ }^{2}$, il ne s'agit pas d'épisodes négligeables. Quand, entre 1927 et 1928, une très jeune Rachel commence à s'essayer à l'écriture, l'une de ses toutes premières tentatives est un texte pour le théâtre, Minha Prima Nazaré. La pièce, jugée par l'auteur elle-même «muito ruim » (apud ARÊAS, 1997, p. 87), ne sera jamais publiée ni représentée. Son existence révèle toutefois un intérêt précoce pour la scène et pour les modalités expressives qu'elle présente.

Rachel de Queiroz ne revient à la dramaturgie qu'en 1952, en qualité de traductrice de la pièce de A. J. Cronin intitulée Os deus riem et publiée par José Olympio. Cette expérience semble lui redonner le goût de la scène: l'année suivante, la première de Lampião a lieu sur la scène du Théâtre Municipal de Rio de Janeiro et ensuite au Teatro Leopoldo Fróes à São Paulo. Grace à cette 
${ }^{3}$ Rachel de Queiroz, abandonnant pour quelques lignes le rôle de dramaturge pour devenir narratrice, donne au metteur en scène des indications scénographiques assez détaillées «Primeiro Quadro, Cena Única. A casa do sapateiro, em ponta de arruado, numa vila à margem do São Francisco. No canto à direita, a parede de frente da casa de taipa, na qual se apóia o telhado do alpendre.[...] O cenário do fundo é o mais simplificado possível. Basta que sugira o arruado, a vila sertaneja, caminho que traz à porta do sapateiro. Sol forte. Sombra só no alpendre. » (QUEIROZ, 2005, p. 13)

\section{Giulia Manera}

dernière mise en scène, la pièce reçoit le prix Saci, attribué par le quotidien $O$ Estado de S. Paulo.

En 1957, Rachel de Queiroz publie chez José Olympio celle qui sera sa deuxième et dernière pièce, A Beata Maria do Egito. Le drame est représenté pour la première fois au Teatro do Serrador de Rio de Janeiro et le rôle de la protagoniste est interprété par Glauce Rocha.

En 1997, en réponse à une question sur la raison de son abandon de la dramaturgie après A Beata Maria do Egito, Rachel de Queiroz affirme simplement: «Eu [...] disse que sou preguiçosa. Eu não escrevi mais para teatro porque ninguém me obrigou » (1997, p. 35). Au de-là de cette paresse déclarée, le maigre succès auprès du public et de la critique ont vraisemblablement contribué à éloigner l'auteur de la scène d'une façon définitive. Sans être tout à fait négatives, la plupart des critiques se limitent à apprécier le style de la dramaturgie de Rachel de Queiroz : mais l'élégance de l'écriture et les dialogues parfaitement orchestrés ne semblent pas parvenir à suppléer à une fragilité de la structure dramatique. Nées pour le théâtre, les pièces Lampião et A Beata seraient ainsi incapables de tenir la scène, et la critique semble suggérer à Rachel de Queiroz - de façon plus ou moins explicite - de continuer à écrire des romans et des chroniques plus que du théâtre.

\section{Lampião ou l'histoire de Maria Bonita}

Dans l'intention de l'auteur, son premier texte pour le théâtre devait s'intituler Maria Bonita. Mais, en l'écrivant, « [...] o Lampião foi crescendo, tomando força e a peça acabou sendo a história dele » (QUEIROZ apud MARTINS, 1997, p. 84). Dans cet acte unique en cinq scènes, le personnage de Maria Bonita, seule femme de la pièce, garde toutefois un poids majeur et, dès la levée du rideau, c'est elle, la dona do rei do cangaço, qui catalyse l'action et donne une cohérence dramatique aux événements.

L'action de la première scène se passe dans un village sertanejo sur les marges du fleuve São Francisco, selon les indications de l'auteur qui tient à dessiner un cadre géographique très précis de l'action ${ }^{3}$. Le décor est simple : une maison, un homme qui travaille tranquillement assis sous le porche, la lumière forte du soleil. Rompant cet équilibre, Maria Déa, une très jolie jeune femme métisse, fait irruption sur scène annonçant l'arrivée de Lampião et de ses cabras dans le village. La peur et la passivité de l'homme, qui l'invite aussitôt à rentrer dans la maison pour broder, se reposer 
et prendre soin des enfants, déclenche une réaction colérique de Maria : elle accuse alors son mari de n'être pas un homme digne de ce nom et lui avoue d'avoir envoyé un message au bandit en lui proposant de l'accueillir dans sa bande. Maria Déa a pris une décision désormais irrévocable: «Disse e faço. Sou bem capaz de ganhar o mundo » (QUEIROZ, 2005, p. 19)

Avant même l'apparition de Lampião, c'est elle qui a donc choisi d'abandonner non seulement son mari, mais aussi la demeure familiale et les enfants. L'action se passe très rapidement, le cangaceiro arrive et Maria le suit, spontanément et sans hésiter, sans même dire adieu à ses fils. La première scène se termine ainsi sur le départ de la femme : la métamorphose de la protagoniste est déjà complète et à partir de ce moment elle cesse de s'appeler Maria Déa pour devenir Maria Bonita.

Dans les scènes suivantes, Rachel de Queiroz nous montre, en un crescendo dramatique, des fragments de la vie quotidienne du Capitão Virgulino, de Maria et des bandits, jusqu'au moment de leur mort dans la grotte do Angico.

L'auteur, qui affirme avoir voulu suivre de près la légende, les anecdotes et les nouvelles transmises par les journaux, semble intéressée plus à la narration de l'humanité de ses personnages qu'à l'action elle-même et aux faits historiques. Fidèle à son écriture, Rachel de Queiroz utilise un style très sobre et mesuré qui lui permet d'éviter toute complaisance dramatique. Scène après scène, la pièce Lampião conserve un réalisme concis qui semble réfléchir le paysage du Nordeste, bien que l'auteur ne cherche pas à représenter de façon réaliste les événements ni à les expliquer. Elle n'innocente pas Virgulino de ses crimes, elle ne se préoccupe pas non plus d'analyser les raison profondes, au niveau sociopolitique, du phénomène du cangaço. Rachel de Queiroz représente simplement "a seqüência de maior dramaticidade [na vida de Lampião] e no-la projetou de um modo quase objetivo. Para tanto, sacrificou os possíveis efeitos que teria alcançado apelando para o pitoresco, mas ganhou uma profundidade rara” (MILLIET, 1989, p. 7)

La scène peut devenir ainsi une fenêtre sur l'univers intime des protagonistes : Rachel de Queiroz représente minutieusement le caractère plus que les gestes hérö̈ques des personnages, explicitant la structure psychologique de Lampião et de Maria Bonita. Dans l'opinion de Sábato Magaldi, la pièce est plus un drame qui parle de l'amour et des passions humaines qu'un récit sur les cangaceiros: 
${ }^{4}$ Avec sa première mise en scène, la pièce A Beata Maria do Egito gagne le Prix "Melhor Peça Teatral" de l'Association des Critiques de Théâtre de São Paulo (1957), le Prix du Théâtre de l'Institut National du Livre (1957) et aussi le Prix Roberto Gomes Livre (1957) et aussi le Prix Roberto Gomes attribue par le Secrétariat de l'Education de Rio de Janeiro (1957). Au delà des reconnaissances officielles, le texte connaît aussi un certain nombre de mises en scènes : l'une des dernières est représentée au Teatro dos Grandes Atores, dans la ville de Barra, Rio de Janeiro, en 1997.
Compreendendo que seria ridículo reconstruir a personalidade de Lampião com as cenas que lhe deram a fama, a autora supõe na peça a sua grandeza, impregna a atmosfera dos cinco quadros do halo que respiramos na lenda, e traz para o macrocosmo do drama a mesma força que existe na realidade acreditada. [...] As cenas de ação de Lampião, fora os diálogos de amor com Maria Bonita, servem [...] para completar-lhe a estrutura psicológica, e não para esboçá-la inicialmente. Esse tratamento só foi possível porque Rachel de Queiroz moveu a trama em torno do amor. (MAGALDI, 1997, p. 263)

$\mathrm{Si}$ on peut partager l'opinion de Magaldi en ce qui concerne l'importance donnée aux personnages plus qu'à l'action, une analyse de la pièce dans la perspective du récit dramatique de Rachel de Queiroz, amène à redimensionner la fonction de l'amour. Le sentiment qui lie les deux protagonistes est indispensable à l'économie de la structure narrative, mais l'auteur ne s'intéresse pas vraiment à la représentation de la relation amoureuse, en pleine cohérence avec une écriture qui évite tout recours à un romantisme facile. L'amour est plutôt l'instrument qui permet d'analyser la personnalité des protagonistes et de montrer les faits par une perspective intime, mineure et inédite -parallèle à la narration des événements historiques. Scène après scène, Rachel de Queiroz représente ainsi les modifications que les personnages vivent en conséquence de leur rencontre : si Maria Déa abandonne sans hésiter son nom, son mari et ses enfants pour le suivre, Lampião devient moins brutal et dans la deuxième partie de la pièce « chega amando, gozando e querendo bem » $(2005$, p. 33$)$. Ce sont donc davantage les conséquences de la relation entre les protagonistes que leur relation en elle-même qui crée la tension dramatique dominante sur scène : la présence de Maria Bonita et les sentiments qu'elle suscite ont en effet la fonction de déstabiliser l'équilibre du groupe des cangaceiros, ce qui amène les spectateurs à pressentir le final tragique. Aux yeux de ses hommes, Lampião semble affaibli par l'amour et Ponto-Fino, son frère, accuse ouvertement Maria Bonita d'être l'origine de ce changement: «Ele diante de você parece mesmo que não tem ação. Todo o mundo não diz que Lampião anda vivo e morto no rabo da sua saia?».

Dans la scène finale, Rachel de Queiroz montre la bande traquée par le gouvernement, décimée par les affrontements et par un Lampião paranoïaque et violent qui en arrive à tuer son propre frère par crainte d'une trahison. Seule Maria Bonita semble consciente du destin qui les attend et Rachel de Queiroz concentre 
${ }^{5}$ On se réfère ici notamment aux opinions de Maria Jacinta dans la Revista da Leitura (novembre 1959), de Henrique Oscar dans le Diário de Notícias (28 octobre 1959) et de Bárbara Heliodora dans le Jornal do Brasil (11 mai 1958). Tous ces articles son cités et analysés par Angela Harumi Tamaro cites et antyses par Angela Hanu 77-78. Le dans sa these de doctorat, pages 77-78. Le document est disponible en ligne, à l'adresse

http://cutter.unicamp.br/document/?code=vt 1s000317235. [consulté le 28 août 2011]

${ }^{6}$ A propos des acceptions du mot beata dans la langue portugaise, il semble intéressant de reprendre la définition qu'en intéressant de reprendre la définition qu'en donne la version de 2001 du Dictionnaire électronique Houaiss, qui rend bien toutes les nuances de sa signification : «1 1 mulher beatificada pela Igreja 2 (1679) mulher extremamente dedicada às práticas religiosas 3 ant. mulher que vivia como freira sem haver ingressado em ordem religiosa [..]" mais aussi sa dimension religiosa $[\ldots]$ " me mais aussi sa dimension péjorative « $[\ldots]$ mulher que afet religiosidade por um comportamento puritano exagerado, freqüentando em excesso missas, rezas e igrejas $[\ldots]$ ». sur elle l'élément tragique qui domine le dernier dialogue avec Lampião:

Maria Bonita $-[\ldots]$ vai haver mais morte, mais inimigo, mais correria... Pois eu, de mim, já me cansei de guerra e sangue. Já me enjoei de andar fugitiva, feito bicho bravo acuado pelos cachorros. [...] Por que a gente não larga desta vida perigosa, não se afunda por esse mundo de meu Deus, lá onde [...] nunca se tinha ouvido falar no nome de Lampião...

Lampião - Só mesmo idéia de mulher! E o que era da minha fama? Já pensou nas mentiras que os "macacos" iam espalhar? [...] que Lampião fugiu com medo da policia e se escondeu [...] ?

Maria Bonita - O que eu sei é que esta nossa vida não pode mais durar.

Par ces paroles, la tension dramatique se révèle dans l'incommunicabilité et la distance qui sépare Maria Bonita et Lampião : si elle est prête à tout abandonner pour continuer à vivre à vivre avec lui-, l'homme, exclusivement préoccupé de sa gloire et de son nom, est disposé à renoncer à tout pour le défendre. L'amour non seulement a assumé la fonction de medium - qui humanise les protagonistes, sans courir le risque de les mythifier -, mais il permet aussi à Rachel de Queiroz de remettre le personnage féminin au centre de la scène. Maria Bonita - désobéissante, héroïque mais profondément et tragiquement humaine -, peut ainsi sortir du second rôle dans lequel l'histoire l'a reléguée et retrouver une voix propre.

\section{Maria d'Egypte, Maria du sertão}

En 1957, quatre ans après la publication de Lampião, Rachel de Queiroz revient à la dramaturgie avec la pièce A Beata Maria do Egito, représentée la même année au Théâtre Serrador de Rio de Janeiro. Pour jouer le rôle titre, l'actrice Glauce Rocha, figure célèbre du théâtre brésilien des années soixante. Le texte rencontre un certain succès auprès du public et reçoit des prix importants ${ }^{4}$, mais les réactions de la critique sont controversées. Comme pour son précédent texte pour la scène, l'élégance et la sobriété de la langue sont reconnues comme le vrai point fort de la pièce. Dans l'opinion de la critique, le texte dans son ensemble manquerait de théâtralité et la voix de la journaliste et de la narratrice résonnerait avec trop d'insistance sur la scène où l'action, expliquée plus que dramatisée, semble perdre son dynamisme ${ }^{5}$.

Dans la pièce, en trois actes et quatre scènes, Rachel de Queiroz raconte l'histoire de Maria, une jeune femme qui a consacré sa vie à Dieu et à la pénitence. Elle est une beata, appellation qui 
${ }^{7}$ Définition de Rachel de Queiroz ellemême (QUEIROZ; QUEIROZ, 1998, p 227). dans la culture brésilienne évoque une femme qui mène la vie d'une religieuse sans toutefois être entrée en religion ou appartenir à un ordre ecclésiastique ${ }^{6}$. Maria, suivie d'un groupe de pèlerins, se dirige vers la ville de Juazeiro da Cruz pour venir en aide au Padre Cicéro, assiégé par les troupes du gouvernement. Au cours du voyage, elle est arrêtée par la police et la pièce se passe entièrement dans le poste de police d'une petite ville du Nordeste, où elle est incarcérée.

Comme pour Lampião, la pièce est inspirée par un fait historique, le siège de la ville de Juazeiro en 1914. L'épisode est particulièrement représentatif dans l'histoire du Nordeste du Brésil car non seulement il montre les tensions entre les oligarchies qui dominent la région au début du $\mathrm{XX}^{\mathrm{e}}$ siècle, mais il fait aussi partie du vécu familial et personnel de Rachel de Queiroz. Le Padre Cicéro était en effet son parrain et un amigo político $^{7}$ de son père, et l'auteur lui dédie un chapitre entier de sa biographie Tantos Anos, dans lequel les faits de Juazeiro sont racontés en détail:

Franco Rabelo não aceitou o governo paralelo do padre Cícero e mandou a tropa. [...] Fez-se o cerco a Juazeiro e, lá dentro, a população entrincheirou-se; mulheres e crianças. Quem não tinha arma, endurecia ao fogo um pão pontiagudo para fazer uma lança ou um chuço. Acorria em defesa do Santo a população de toda a região, fanáticos, beatos, jagunços. Juazeiro era chamado a Nova Jerusalém. As tropas do governo foram vencidas e se retiraram. (QUEIROZ; QUEIROZ, 1998, p. 234)

Si cet épisode, à la fois historique et épique, représente la circonstance de la pièce, c'est toutefois la figure de sainte Marie d'Egypte qui inspirer directement le personnage de la beata. Comme pour la sainte chrétienne, la Maria nordestine utilise son corps comme monnaie d'échange pour retrouver la liberté et reprendre son voyage. La matière, qui combine donc réalité historique, religiosité populaire et hagiographie chrétienne, devient pour Rachel de Queiroz un instrument pour analyser et raconter sur scène la personnalité des protagonistes. Tout en étant des figures prototypiques - la beata, le tenente de police, le colonel qui incarne le pouvoir politique et le cabo, soumis et superstitieux, qui représente la voix du peuple - ces personnages ne deviennent jamais des masques tragiques, mais restent des individus avec une forte humanité. C'est cette humanité qui crée la tension dramatique qui domine la pièce: Rachel de Queiroz n'a aucune volonté moralisatrice, elle ne veut ni expliquer les faits ni donner 
${ }^{8}$ On identifie ici la première production narrative de Rachel de Queiroz avec les romans - O Quinze, João Miguel, Caminho de Pedras, As Três Marias - publiés entre le 1930 et le 1939 et la deuxième avec Dôra, Doralina et Memorial de Maria Moura, Doralina et Memorial de Maria Moura, de 1992. Le folheto Galo de Ouro, publié chaque semaine sur la revue "O Cruzeiro" a partir de 1950, semble être peu représentatif d'un discours sur la figuration du féminin de l'auteur et n'est donc pas pris en compte dans notre travail.

${ }^{9}$ Une dualité utilisé par Luís Bueno pour décrire la différence de Conceição protagoniste de $O$ Quinze (2006, p. 286) mais qu'on retrouve aussi dans l'article de Rachel de Queiroz "A imagem féminina" (publié dans $O$ Estado de Minas - le 11 juin 2000. L'article est consultable sur le site de l'Académie Brésilienne de Lettres, à l'adresse :

http://www.academia.org.br/abl/cgi/cgilua.e xe/sys/start.htm?from_info_index=26\&infoi d=2858\&query=advsearch\&search_by_auth orname=Rachel+de+Queiroz\&search_by_fi eld=tax\&search_by_headline=false\&search by keywords=any\&search by priority=all \&search by section=all\&search by state $=$ all\&search text options $=$ all\&sid- $=438 \&$ text $=$ l'absolution à ses personnages, mais simplement raconter leur labeur intérieur avec la sobriété qui est propre à son écriture.

Si, selon Sábato Magaldi, la pièce raconte un « caso de amor muito humano » $(1997$, p. 264) ou, pour Paulo Rónai, l'histoire de la Sainte Marie d'Egypte présente une forte suggestion romantique (1989, p. 11), Rachel de Queiroz toutefois ne semble pas vouloir raconter ou représenter la relation entre Maria et le Tenente. Avec un processus similaire à celui qu'elle utilise pour Lampião et Maria Bonita, l'auteur se sert du sentiment qui lie - ou éloigne - les protagonistes pour analyser les réactions qu'il déclenche dans leurs personnalités respectives, afin de les faire émerger avec une force dramatique majeure. Comme un virus inoculé dans deux cobayes, le sentiment agit sur Maria et sur le Tenente : observer les effets opposés qu'il produit sur eux devient pour Rachel de Queiroz le moyen d'en comprendre le caractère et d'approfondir la psychologie, bien plus qu'un simple recours narratif.

\section{La beata et la cangaceira: figurations du féminin dans} l'écriture de Rachel de Queiroz

Femme qui écrit sur des femmes, Rachel de Queiroz contribue de façon déterminante à l'écriture de l'imaginaire féminin et, au- delà, inaugure une quête identitaire qui sera centrale dans l'écriture féminine à partir des années soixante. Les deux textes théâtraux en analyse, dans lesquels les personnages féminines se placent directement au centre de la scène et de l'action dramatique, permettent de définir la figuration du féminin qui Rachel de Queiroz construit depuis son premier roman.

Si on analyse attentivement les personnages de Maria Bonita et de Maria do Egito, on retrouve de nombreuses analogies avec les figures féminines imaginées par l'auteur au long de sa carrière. Dans cette perspective, il est possible d'affirmer que les deux Maria théâtrales occupent une place non négligeable dans la galerie de portraits de femme réalisés par Rachel de Queiroz: représentantes d'une généalogie de femmes autonomes et atypiques, les deux Maria sont capables d'incarner une rupture totale avec les espaces et les rôles traditionnellement réservés aux femmes dans la société. C'est cette différence qui les met en parfaite cohérence avec les autres images féminines de l'écrivaine. $\mathrm{Au}$ de-là de cette affirmation, il est possible de considérer aussi que ces deux personnages, imaginés entre 1953 et 1957, représentent une étape fondamentale dans la figuration du féminin de Rachel de Queiroz. Le deux Maria théâtrales se situent en effet entre la première et la 
deuxième production romanesque de l'auteur ${ }^{8}$, non seulement d'un point de vue chronologique, mais aussi pour leurs caractéristiques. Si Conceição, Noemi ou encore Guta, sont encore des personnages « em transito », partagés entre tradition et modernité, maison ou rue, respect ou rupture de la norme sociale, acceptation ou revendication de soi, Maria Bonita et Maria do Egito sont deux figures bien plus radicales. Elles ont abandonnée d'une façon définitive et irréversible l'espace clos de la maison et le rôle traditionnel de mères et épouses pour ganhar o mundo et affirmer leur choix. La beata et la cangaceira incarnent ainsi un «sujeito desviante da norma » (XAVIER, 1998, p. 37) qui refuse le destin de femme et se soustrait à la dichotomie fiancée/prostituée ${ }^{9}$ récurrente dans la littérature brésilienne de la première moitié $\mathrm{du} \mathrm{XX}^{\mathrm{e}}$ siècle. Dans leur désobéissance, ces personnages semblent anticiper Maria Moura, la dernière et la plus radicale des femmes imaginées par Rachel de Queiroz.

Abandonner la maison, se soustraire au diktat du mariage et de la maternité - par le cangaço ou la croyance religieuse représentent des moments différents d'une égale négation du féminin socialement et culturellement déterminé. La bandit et la sainte incarnent ainsi, comme feront quelques années après Dôra ou
Maria Moura, une alternative : affirmer leur différence signifie vivre en marge de la société, l'une hors la loi, traquée par la police, l'autre dans la solitude d'une sainteté autoproclamée.

Dans sa lecture de Dôra, Doralina et Memorial de Maria Moura, derniers romans de l'écrivaine, Wilson Martins parle de « temática feminista » $(1997$, p. 83), une définition qui est possible attribuer aussi aux textes pour le théâtre de Rachel de Queiroz. Avec les pièces Lampião et A Beata Maria do Egito, l'auteur représente deux personnages féminins qui ont choisi leur destin librement. La vie de Maria Bonita n'est en rien le fruit du hasard ou la conséquence des sentiments pour Virgulino mais, au contraire, c'est le produit d'un choix rationnel et revendiqué en tant que tel. Dans la première scène, Maria Déa affirme d'être déterminée à tout abandonner : «Disse e faço. Sou bem capaz de ganhar o mundo » (p. 19). Ce n'est pas donc l'amour pour un homme qu'elle ne connaît même pas qui motiver son choix, mais plutôt la volonté de ganhar o mundo. Une volonté que la protagoniste réaffirme dans la quatrième scène : «Eu levo esta vida porque quero » (p. 88).

Comme Maria Bonita, Maria do Egito - appelée dans la pièce « santa cangaceira » (p. 164), ce qui rend le parallélisme encore plus évident - n'est en aucun moment une victime: elle aussi a 
abandonné la maison de sa propre volonté, elle a choisi librement de se vouer à l'ascétisme, la prière et la défense des pauvres. Dans la pièce sa détermination, qui frise le fanatisme, devient le centre de l'action dramatique : Maria reste totalement indifférente à l'amour que le Tenente éprouve pour elle, un amour total qui le conduira à la mort. Le péché, ainsi que le sentiment, la traverse «como sol passando pela vidraça » (p. 178) : la beata est bien déterminée à ne pas être sauvée d'une vie qu'elle a choisie et qu'elle revendique.

Maria do Egito et Maria Bonita, dans leur différence même, sont deux figures féminines également radicales, non conformes et héroïques en tant que telles. C'est cette exceptionnalité, cette intransigeance que Rachel de Queiroz veut raconter : elle ne semble pas s'intéresser à une description de femmes dans leur simple quotidienneté, ni à représenter ou à dénoncer par l'instrument de son écriture la subordination ordinaire de leur vie, enfermées à la maison dans le rôle ancien d'épouse et génitrice. Il s'agit d'un thème central de son écriture, ainsi qu'elle l'affirme dans une note du programme de la pièce A Beata Maria do Egito, pour sa représentation au Teatro Serrador de Rio de Janeiro:

\begin{abstract}
A minha idéia deu corpo a um tema que me interessara sempre, que eu já tentara mais de uma vez em outras experiências e em diferentes situações: o comportamento da criatura que a si considera excepcional (que se considera um santo, por exemplo), posta dentro da correnteza de paixões e conflitos em que se debatem os outros mortais. (1989, p. 13)
\end{abstract}

Sur la scène comme sur la page, Rachel de Queiroz imagine ainsi une véritable galerie de femmes exemplaires et héroïques qui revendiquent la liberté de choisir son propre destin.

\section{Hérö̈nes féminines et femmes héroïques}

Quand, pendant la décennie de 1950, Rachel de Queiroz écrit pour le théâtre, elle veut surtout raconter les histoires de deux femmes qui, dans un sertão à la dimension à la fois historique et mythique, revendiquent un choix de vie radicale et irréversible. Mais Maria Bonita et Maria do Egito ne sont pas des figures neutres, des cadres vides. Si les protagonistes de son œuvre narrative n'étaient sorties que de son imagination, avec les Maria théâtrales Rachel de Queiroz se trouve confronté à une image prédéfinie aux contours bien nets. Plus qu'à un processus d'inventio, l'auteur doit donc se consacrer la représentation : elle s'approprie le personnage 
et lui restitue sur scène une voix, un corps, une tridimensionnalité qu'il ne peut plus avoir sous son aspect historique et mythique.

Pour représenter Maria Bonita et Maria do Egito, l'auteur doit donc dialoguer avec l'image qu'elles évoquent et la rendre cohérente avec ses intentions narratives et dramatiques. Mais de quelle manière Rachel de Queiroz s'approprie-t-elle de ces hérö̈nes féminines et travaille leurs personnages pour les rendre crédibles ? Comment montrer leur humanité sur scène, sans les caricaturer ou dénaturer leur image ?

Les représentations des deux Maria, solidement enracinées dans l'imaginaire du Nordeste du Brésil, sont en effet le produit de la sédimentation de plusieurs éléments historiques, religieux, populaires et littéraires, qui proviennent tant de la tradition littéraire brésilienne que de l'européenne. Lire la stratigraphie de significats et de suggestions qui est à la base des personnages féminins de Lampião et de A Beata Maria do Egito permet d'analyser le processus d'écriture de Rachel de Queiroz et de mieux comprendre ses intentions.

Quand Rachel de Queiroz imagine Maria Bonita, elle se confronte à une image hautement symbolique, capable d'incarner le thème du banditisme féminin, bien présent dans la culture et l'imaginaire du Nordeste du Brésil. Il s'agit d'un argument récurrent dans son œuvre et que Rachel de Queiroz utilise pour une représentation du pouvoir dans sa déclinaison féminine: la cangaceira, comme la matriarche, occupe une place et un espace le sertão, la rue - traditionnellement réservée aux hommes et tente de définir un « espaço de domínio próprio » (HOLLANDA, 1997, p. 107). Figure littéraire bien nordestine, la femme bandit est avant tout un personnage historique réel, comme la Maria Oliveira - une autre Maria, une autre héroïne - que Rachel de Queiroz découvrit par hasard lors de recherches pour un livre:

Eu estava fazendo um trabalho com minha irmã Maria Luiza sobre a seca do Nordeste. [...] Descobrimos que a primeira grande seca registrada oficialmente aconteceu em Pernambuco em 1602. Nesta seca, uma mulher chamada Maria de Oliveira tornou-se conhecida, porque, juntamente com os filhos e uns cabras, saiu assaltando fazendas. Pois eu fiquei com essa mulher na cabeça. Uma mulher que saía com os filhos e um bando de homens assaltando fazendas - era a "Lampiona" da época, pense (Cadernos, 1997, p. 34). 
${ }^{10}$ Maria Moura, protagoniste du Memorial de Maria Moura de 1992, est le dernie personnage féminin imaginé par Rachel de Queiroz Dans ce roman, l'écrivaine raconte la vie d'une jeune femme qui, après la mort la vie d'une jeune fenme qui, après la mor de ses parents, est obligée à abandonne d'une façon rocambolesque sa maison et sa fazenda, pour protéger sa vie et sa liberté. Obligée à une fuite dans le sertão, Maria Moura décide de réagir et devient le chef d'une bande de cangaceiros, redoutée et respectée. Bien que plus radical, ce personnage montre nombreuses analogies persona analogies avec la Maria Bonita les deux femmes renoncent de façon définitive a leur passé, changent leur nom et choisissent de ganhar o mundo. Comme Maria Bonita, Maria Moura n'est en aucun moment une victime, mais elle revendique et assume ses choix

\section{Giulia Manera}

Femme et cangaceira, Maria Bonita peut être lue en tant que première incarnation de Maria Moura ${ }^{10}$ : les recherches faites par Rachel de Queiroz à l'occasion de l'écriture de Lampião constituent en effet un matériel que l'auteur va reprendre pour donner forme à la figure principale de son ultime œuvre romanesque. Les analogies sont évidentes et bien plus profondes que puissent paraitre à une toute première lecture. Comme Maria Moura, Maria Bonita:

É uma bandoleira gerada pelas disputas de terra do sertão, cujo modelo encontramos na tradição peninsular la literatura de cordel que aportou no Nordeste brasileiro. [...] ao bandoleiro [...] substitui-se sua representante feminina, que pode ser tratada de maneira burlesca, ou de modo dramático (ARÊAS, 1997, p. 100)

Le personnage féminin de la pièce Lampião est le résultat d'un processus de dramatisation: l'auteur utilise les références au modèle historique et littéraire comme cadre narratif dans lequel elle peut représenter l'intériorité de l'individu au centre de la scène. Le langage du burlesque et de la parodie ne se retrouve en aucun moment dans la production théâtrale de Rachel de Queiroz.
$\mathrm{Si}$, pour donner forme à la Beata, l'auteur adopte le même processus de dramatisation des modèles identifié dans sa première pièce, elle dialogue maintenant avec un système de références plus multiforme encore.

Comme Maria Bonita, et davantage encore, la figure de Maria do Egito est le résultat d'une sélection et d'une harmonisation d'éléments très différents avec lesquels Rachel de Queiroz compose un texte évocateur, capable de combiner plusieurs suggestions. Dans le cas de la Beata, la référence la plus évidente est sans doute celle de la figure de la sainte chrétienne. Appartenant à l'hagiographie, la Sainte Marie d'Egypte arrive au Brésil grâce au livre liturgique Lendas Douradas, une vulgarisation du Flos Sanctorum, le principal recueil des vies des saintes. C'est grâce à ce livre, lue par sa grandmère, qu'une Rachel de Queiroz encore enfant découvre l'histoire de la sainte égyptienne. Au souvenir d'enfance et à l'image des nombreuses femme beatas qui circulent dans la région, vont s'ajouter les lectures classiques et les interprétations que la culture populaire donne de cette figure : la martyre chrétienne est non seulement un personnage inspirateur des écrivains et poètes, de Rilke à Manuel Bandeira, mais aussi la protagoniste du folheto de cordel portugais Auto de Santa Maria Egipcíaca. Il s'agit d'un 
système de références multiforme et complexe que Rachel de Queiroz assume et tient à expliquer au public qui s'apprête à assister à la pièce. En occasion de la première mise en scène de $A$ Beata Maria do Egito au Teatro do Serrador à Rio de Janeiro, l'auteur écrit en effet le texte déjà cité História de Beata qui explique les sources d'inspiration de l'œuvre. Il s'agit d'une sorte de captatio benevolentiae utile pour éclairer le processus d'écriture de la pièce et les intentions qui le motivent:

Tomei como ponto de partida uma velha lenda cristã (Santa Maria Egipcíaca) que sempre me invocara, e que depois de posta em balada por Manuel Bandeira [...] tomara formas de fascinante beleza e crescera mais na sua sedução misteriosa. [...] Fixara-me numa Maria Egipcíaca nordestina, uma daquelas beatas de hábito de freiras que outrora pululavam pelo Cariri; dei-lhe o nome de Maria do Egito - única analogia direta que me permiti com a santa verdadeira [...] Minha esperança é que, posta a funcionar no palco, A Beata se liberte de mim [...]. E assim veremos se voa mesmo [...] a minha oscura lagarta da serra da Mombaça, filha longínqua do Flos sanctorum da minha avó, de uma balada de Manuel, lembrança de figuras vistas na infância. Santa frustrada, irmã enjeitada de cangaceiros e fanáticos - por ela peço passagem e imploro complacência (QUEIROZ, 1989, p. 13-15).
Comme dans le cas de Maria Bonita, pour donner forme à la protagoniste de sa deuxième pièce et l'humaniser, Rachel de Queiroz procède à un dramatisation des éléments traditionnels. Le modèle historique et littéraire est donc élaboré dans une perspective analytique et existentielle. Rachel de Queiroz travaille ainsi la substance, l'intériorité de cette figure - une femme, une beata, une héroïne - plus que la forme: sa préoccupation n'est jamais exclusivement esthétique. Maria do Egito, exactement comme Maria Bonita, représente ainsi pour l'auteur l'occasion et l'instrument pour poursuivre ce discours sur l'altérité féminine, un discours commencé avec $O$ Quinze et jamais interrompu. Rachel de Queiroz, par sa maestria, arrive à faire de la beata une figure réaliste et profondément humaine, sans jamais rendre caricaturale l'appropriation des données historiques ou littéraires. Dans cette perspective, il est possible d'affirmer que, sur la scène les deux Maria, héroïnes féminines nordestines par excellence, peuvent finalement exprimer leur individualité, leur humanité et devenir des femmes héroïques. Héroïques car exceptionnelles, différentes : elles défient ouvertement le gouvernement et les oligarchies, désobéissent, refusent le rôle de victime et restent cohérentes avec leur choix, jusqu'aux extrêmes conséquences. 
11 On reprend ici le discours sur l'émergence d'une dramaturgie féminine au Brésil proposé par Elza Cunga de Vincenzo (1992, p. 16). Les œuvres de Prado et
Magaldi sont citée dans notre bibliographie.

${ }^{12}$ C'est l'époque de la publication de $O$ Galo de Ouro, le roman décidément le plus "carioca" de l'auteur qui, à cette époque, compose des chroniques liées essentiellement au quotidien et à la vie à

Rio de Janeiro.

\section{Giulia Manera}

\section{Rachel de Queiroz dans la dramaturgie brésilienne de la} décennie de 1950

Si l'écriture théâtrale ne représente qu'un épisode circonscrit d'une longue carrière consacré au roman et au journalisme - et la critique est presque unanime à voir dans la dramaturge une Rachel de Queiroz mineure-, une fois reconnue la profonde cohérence de cette écriture avec le discours narratif de l'auteur, il semble intéressant d'analyser Lampião et A Beata Maria do Egito dans le contexte de la dramaturgie brésilienne de la deuxième moitié du $\mathrm{XX}^{\mathrm{e}}$ siècle.

Dans un article pour "O Estado de São Paulo" commentant l'exorde de Rachel de Queiroz comme dramaturge avec Lampião, Sérgio Milliet affirme que pour l'auteur la vocation de l'écriture théâtrale est une conséquence directe de l'essor considérable que le théâtre connaît en ce moment au Brésil:

Rachel de Queiroz sentiu-se agora atraída pelo teatro. Não é de estranhar, pois seus romances e contos sempre se revelaram de ação muito intensa e se caracterizaram pela naturalidade dos diálogos. Se não tentou antes escrever peças, foi sem duvida porque somente nos últimos anos se tornou o teatro brasileiro uma realidade. Hoje ele tem público, atores e cenaristas. E as obras vão afluindo (MILLIET, 1989, p. 7).
Même d'une façon épisodique, Rachel de Queiroz participe donc à la naissante dramaturgie moderne brésilienne. En tant que femme qui écrit pour la scène, elle se distingue comme une pionnière entre les pionniers : si on consulte les œuvres dédiés à l'histoire du théâtre brésilien - à partir de celles de Décio Almeida Prado et de Sábato Magaldi - il est possible en effet de constater l'absence d'auteurs femmes, au moins jusqu'à la fin de la décennie de $1960^{11}$. Rachel de Queiroz, en tant que dramaturge, est donc pour le théâtre brésilien des années Cinquante, dans une moindre mesure, la desbravadora qu'elle avait été pour le roman dans les année Trente. L'une des premières femmes à écrire pour la scène dans le pays, elle partage avec les dramaturges qui sont en train de réformer le théâtre brésilien non seulement une proximité chronologie, mais surtout des intentions et des thématiques similaires.

Bien que, dans les années 1950, l'auteur soit devenue une carioca d'adoption, ses pièces sont inspirées par la tradition et la culture régionale du Nordeste, bien plus que les chroniques ou le roman qu'elle écrit au même moment ${ }^{12}$. Le caractère fortement régionaliste de la production théâtrale de Rachel de Queiroz émerge aussi à une lecture de l'entretien réalisé pour le Cadernos de Literatura Brasileira, de l'Institut Moreira Salles: 
Cadernos: Escritores como Jorge Amando e Ariano Suassuna valem-se bastante da tradição popular do Nordeste na produção de suas obras. Em seus romances, diferentemente de seu teatro, isto é pouco frequente. Por quê?

Rachel de Queiroz: Eu uso cenários típicos, discuto problema, mas realmente não sou a regionalista típica. $\mathrm{O}$ Ariano tem aquilo incorporado, [...] é quase um médium da tradição. (1997, p. 26)

Lampião et A Beata révèlent bien des axes thématiques communs à la dramaturgie contemporaine nordestine qui est en train de connaître un grand essor, grâce notamment à l'expérience du Théâtre de l'Etudiant de Pernambouc (TEP) et du Théâtre Populaire du Nordeste (TPN). Fondés respectivement en 1940 et en 1960 par Hermilo Borba Filho et Ariano Suassuna et animés par un groupe de jeunes poètes, acteurs, écrivains et peintres, ces deux Théâtres deviennent le centre d'irradiation de la nouvelle dramaturgie: on recherche la fondation d'un art dramatique national qui reflète les idées, les problèmes et les intérêts du peuple (SANTOS, 2008). Les mythes, les héros et les récits de cette culture régionale deviennent ainsi des éléments fondateurs d'un discours scénique qui vise au mélange de la tradition locale et de la littérature dite érudite, en un complexe mouvement de tissage des sources. Un processus dialectique, pour reprendre la définition de Cândido, qui résulte de la tension entre la donnée locale, qui se présente en tant que substance de l'expression, et les formes héritées de la tradition européenne qui coïncident avec la forme de l'expression (2006, p. 109). Même si les résultats stylistiques sont très différents, la multiplicité des sources, érudites comme populaires, qui inspirent Lampião et $A$ Beata semblent rapprocher ces pièces de la dramaturgie de Ariano Suassuna et de Hermilo Borba. Mais ce sont les thématiques qui révèlent les analogies les plus significatives: pour ne citer que l'exemple le plus évident, la protagoniste de la Donzela Joana de Borba, représentée pour la première fois en 1966, rappelle A Beata de Rachel de Queiroz. Non seulement on peut retrouver dans les deux pièces la transmigration d'un personnage de l'hagiographie ou de la tradition chrétienne au quotidien du Nordeste du Brésil, mais tant Joana que Maria do Egito incarnent la figure de la sainte - plus ou moins guerrière - qui lutte contre la politique des puissants et trouve dans la fois la force de résister. Borba Filho confirme ces analogies et en suggérent d'autres:

Todo o Nordeste é um drama de primeira grandeza, com a tragédia das secas, a escravidão do açúcar e o cangaceirismo. [...] É poesia viva, é poesia explodindo pela boca dos cantadores de 
$\mathrm{ABC}$ das figuras heroicas do sertão, das figuras lendárias de [..] Zumbi dos Palmares, de Lampião. [...] O romance brasileiro já se preocupou com esses assuntos [...]. O teatro sempre se manteve afastado. (BORBA apud PRADO, 2001, p. 79)

Dans les années qui précèdent le coup d'Etat de 1964, le théâtre, spécialement dans le Nordeste, cherche à présenter sur scène des modes de vie anti ou asociales, potentiellement révolutionnaires, comme par exemple le cangaço et le messianisme (PRADO, 2001, p. 99). Modes de vie représentés par des personnages historiques comme Lampião et Padre Cícero, qui inspirent plus ou moins directement les pièces Lampião et A Beata Maria do Egito. Toutes ces considérations contribuent non seulement à contextualiser ces pièces dans l'histoire du théâtre brésilien mais permettent aussi de considérer que Rachel de Queiroz, avec sa diction si personnelle et cohérente, intègre d'une façon épisodique, mais certainement non négligeable, la dramaturgie contemporaine.

\section{Références bibliographiques}

AREAS, Vilma. Rachel: o ouro e a prata da casa. In: Rachel de Queiroz, Cadernos de Literatura Brasileira. Rio de Janeiro: Instituto Moreira Salles, 1997.
BUENO, Luís. Uma história do romance de 30. São Paulo: Editora da Universidade de São Paulo; Campinas: Editora da Unicamp, 2006.

CANDIDO, António. Literatura e sociedade. $9^{\mathrm{e}}$ ed. Rio de Janeiro: Ouro sobre azul, 2006.

HOLLANDA, Heloísa Buarque de. O éthos Rachel. In: Rachel de Queiroz, Cadernos de Literatura Brasileira. Rio de Janeiro: Instituto Moreira Salles, 1997.

MAGALDI, Sábato. Panorama do teatro brasileiro. $3^{\mathrm{e}}$ ed. São Paulo: Global, 1997.

MARTINS, Wilson. Rachel de Queiroz em perspectiva. In: Rachel de Queiroz, Cadernos de Literatura Brasileira. Rio de Janeiro: Instituto Moreira Salles, 1997.

MILLET, Sérgio. Nota introdutória. In: QUEIROZ, Rachel de. Obra Reunida. vol. 5. Rio de Janeiro: José Olympio, 1989.

PRADO, Décio de Almeida. O Teatro Brasileiro Moderno. $2^{\mathrm{e}}$ ed. São Paulo: Editora Perspectiva, 2001. 
QUEIROZ, Rachel de. Lampião, A beata Maria do Egito. $5^{\mathrm{e}}$ ed. Rio de Janeiro: José Olympio, 2005.

QUEIROZ, Rachel de. História de Beata. In: QUEIROZ, Rachel de. Obra Reunida. vol. 5. Rio de Janeiro: José Olympio, 1989.

QUEIROZ, Rachel de; QUEIROZ, Maria Luíza de. Tantos anos. $2^{\mathrm{e}}$ ed. São Paulo: Editora Siciliano, 1998.

Rachel de Queiroz, Cadernos de Literatura Brasileira. Rio de Janeiro: Instituto Moreira Salles, 1997.

RÓNAI, Paulo. A Beata Maria do Egito. In: QUEIROZ, Rachel de. Obra Reunida. vol. 5. Rio de Janeiro: José Olympio, 1989, p. X-XII

SANTOS, Idelette Muzart Fonseca dos. «Ariano Suassuna l'homme, l'oeuvre et ses parcours », Plural Pluriel - revue des cultures de langue portugaise, $\mathrm{n}^{\circ} 1$, printemps-été 2008, [En ligne] URL: www.pluralpluriel.org.

VINCENZO, Elsa Cunha de. Um teatro da mulher: dramaturgia feminina no palco brasileiro contemporâneo. São Paulo: Perspectiva Editora, Edusp, 1992.
XAVIER, Elodia, Declino do Patriarcado: a familia no imaginario feminino. Rio de Janeiro: Rosa dos Tempos, 1998. 